\title{
Optimal receiver antenna location in indoor environment using dynamic differential evolution and genetic algorithm
}

\author{
Shu-Han Liao ${ }^{1}$, Chien-Hung Chen ${ }^{2}$, Chien-Ching Chiu ${ }^{1 *}$, Min-Hui Ho ${ }^{1}$, Tadeusz A Wysocki ${ }^{3}$ and Beata J Wysocki ${ }^{3}$
}

\begin{abstract}
Using the impulse responses of these multipath channels, the bit error rate (BER) performance for binary pulse amplitude modulation impulse radio ultra-wideband communication system is calculated. The optimization location of receiving antenna is investigated by dynamic differential evolution (DDE) and genetic algorithm (GA) to minimize the outage probability. Numerical results show that the performance for reducing BER and outage probability by DDE algorithm is better than that by GA.
\end{abstract}

Keywords: BER; BPAM; UWB; DDE; GA

\section{Introduction}

Ultra-wideband (UWB) transmissions can legally operate in the range from $3.1 \mathrm{up}$ to $10.6 \mathrm{GHz}$ at a limited transmit power of $-41 \mathrm{dBm} / \mathrm{MHz}$ [1]. All wireless systems must be able to deal with the challenges of operating over a multipath propagation channel, where the object in the environment can cause multiple reflections. Bit error rate (BER) degradation is caused by the inter-symbol interference due to a multipath propagation made up of radio wave reflections by walls, ceilings, floors, and office fixtures. In general, effective antenna selection and deployment strategies are important for reducing BER in indoor wireless systems [2,3]. To the best of our knowledge, there is still no investigation using the dynamic differential evolution (DDE) and genetic algorithm (GA) to optimize the location of receiving antennas for minimizing the BER in indoor wireless communication channel.

Based on the BER formula, the outage probability is chosen as the cost function in our optimization procedure. Thus, the optimization of location of receiving antenna in this paper is using DDE and GA to overcome the above situation and to minimize the outage probability.

The remainder of this paper is organized as follows: In Section 2, channel modeling, system description, dynamic

\footnotetext{
* Correspondence: chiu@ee.tku.edu.tw

'Department of Electrical Engineering, Tamkang University, Tamsui District, New Taipei City 251, Taiwan

Full list of author information is available at the end of the article
}

differential evolution, and genetic algorithm procedure are presented. The numerical results are then presented in Section 3, and conclusion is made in Section 4.

\section{Channel modeling and system description}

\subsection{Channel modeling}

The following two steps are used to calculate the multipath radio channel:

1. Frequency responses for sinusoidal waves by shooting and bouncing ray (SBR)/image [4-23] techniques.

The SBR/image method can deal with highfrequency radio wave propagations in the complex indoor environments $[24,25]$.

2. Inverse fast Fourier transform (IFFT) and Hermitian processing. The frequency responses are transformed to the time domain using the inverse Fourier transform with the Hermitian signal processing [26]. Using the Hermitian processing, the pass-band signal is obtained with zero padding from the lowest frequency down to direct current (DC), taking the conjugate of the signal and reflecting it to the negative frequencies. The result is then transformed to the time domain using IFFT [27]. Since the signal spectrum is symmetric around DC. The resulting doubled-side spectrum corresponds to a real signal in the time domain. 
The equation for modeling the multipath radio channel is a linear filter with an impulse response given by

$$
h(t)=\sum_{n=1}^{N} \alpha_{n} \delta\left(t-\tau_{n}\right)
$$

where $N$ is the number of paths observed at time, $\delta(\cdot)$ is the Dirac delta function, and $a_{n}$ and $\tau_{n}$ are the channel gain and time delay for the $n$-th path, respectively.

\subsection{System block diagram}

The block diagram of the simulated communication system is shown in Figure 1. The received signal $r(t)$ can be expressed as follows:

$$
r(t)=[x(t) \otimes h(t)]+n(t)
$$

where $x(t)$ is the transmitted signal, $h(t)$ is the impulse response of the equivalent baseband, and $n(t)$ is the white Gaussian noise with zero mean and variance $N_{0} / 2$.

The transmitted UWB pulse stream can be expressed as follows [28]:

$$
x(t)=\sum_{n=1}^{\infty} p\left[t-(n-1) T_{\mathrm{d}}\right] d_{n}
$$

where $p(t)$ is the transmitted waveform. $d_{n} \in\{ \pm 1\}$ is a binary pulse amplitude modulation (B-PAM) symbol and is assumed to be independent and identically distributed. $T_{\mathrm{d}}$ is the duration of the transmitting signal. The transmitted waveform $p(t)$ is the Gaussian waveform with ultra-short duration $T_{\mathrm{p}}$ at the nanosecond scale. Note that $T_{\mathrm{d}}$ is the duration of the transmitting signal, and $T_{\mathrm{p}}$ is the pulse duration. The value of $T_{\mathrm{d}}$ is usually much larger than that of $T_{\mathrm{p}}$. The Gaussian waveform $p(t)$ can be described by the following expression:

$$
p(t)=\frac{1}{\sqrt{2 \pi} \sigma} e^{\frac{-t^{2}}{2 \sigma^{2}}}
$$

where $t$ and $\sigma$ are time and standard deviation of the Gaussian wave, respectively. The average transmit energy symbol $E_{\mathrm{t}}$ can be expressed as

$$
E_{\mathrm{t}}=\int_{0}^{T_{\mathrm{d}}} p^{2}(t) d t
$$

where $E_{\mathrm{t}}$ is the average transmitted energy.

The correlation receiver samples the received signal at the symbol rate and correlates them with suitably delayed references given by

$$
q(t)=p\left[t-\tau_{1}-(n-1) T_{\mathrm{d}}\right]
$$

where $\tau_{1}$ is the delay time of the first wave. The output of the correlator at $t=n T_{\mathrm{d}}$ is $[29,30]$ :

$$
\begin{gathered}
Z(n)=\int_{(n-1) T_{\mathrm{d}}}^{n T_{\mathrm{d}}}\left\{\left[\sum_{n=1}^{\infty} p\left[t-(n-1) T_{\mathrm{d}}\right] d_{n}\right] \otimes h(t)\right\} \\
\cdot q(t) d t+\int_{(n-1) T_{\mathrm{d}}}^{n T_{\mathrm{d}}} n(t) q(t) d t \\
=V(n)+\eta(n) .
\end{gathered}
$$

It can be shown that the noise components $\eta(n)$ of Equation 7 are uncorrelated Gaussian random variables with zero mean. The variance of the output noise $\eta$ is

$$
\sigma^{2}=\frac{N_{0}}{2} E_{t} .
$$

The conditional error probability of the $n$-th bit is thus expressed by

$$
P_{e}[Z(n) \mid \vec{d}]=\frac{1}{2} \operatorname{erfc}\left[\frac{V(n)}{\sqrt{2} \sigma} \cdot\left(d_{n}\right)\right],
$$

where $\operatorname{erfc}(x)=\frac{2}{\sqrt{\pi}} \int_{x}^{\infty} e^{-y^{2}} d y$ is complementary error function and $\{\vec{d}\}=\left\{d_{0}, d_{1}, \ldots, d_{n}\right\}$ is the binary sequence. Note that the average BER for B-PAM impulse radio UWB system can be expressed as [10]

$$
\mathrm{BER}=\sum_{i=1}^{2^{n}} P(\vec{d}) \cdot \frac{1}{2} \operatorname{erfc}\left[\frac{V(i)}{\sqrt{2} \sigma} \cdot\left(d_{n}\right)\right],
$$

where $P(\vec{d})$ is the occurring probability of the binary sequence $\vec{d}$.

\subsection{Dynamic differential evolution}

In dynamic differential evolution (DDE), after generating the initial population, the candidate solutions are refined by applying mutation, crossover, and selection, iteratively. The flowchart of the DDE algorithm is shown in Figure 2. In this strategy, a mutant vector for each target vector $\mathrm{V}_{j}^{k+1}$ at the $k+1$ generation is computed by

$$
\begin{aligned}
& \left(\mathrm{V}_{j}^{k+1}\right) i=\left(\mathrm{X}_{j}^{k}\right) i+\zeta \cdot\left[\left(\mathrm{X}_{\text {best }}^{k}\right) i-\left(\mathrm{X}_{j}^{k}\right) i\right]+X \cdot\left[\left(\mathrm{X}_{m}^{k}\right) i-\left(\mathrm{X}_{n}^{k}\right) i\right], \\
& j, m, n \in\left[0, N_{\mathrm{p}}-1\right], \quad m \neq n,
\end{aligned}
$$

where $i=1 \sim D$ and $\chi$ and $\zeta$ are the scaling factors associated with the vector differences $\left(\mathrm{X}_{\text {best }}^{k}-\mathrm{X}_{j}^{k}\right)$ and $\left(\mathrm{X}_{m}^{k}-\mathrm{X}_{n}^{k}\right)$, respectively. The disturbance vector $\mathrm{V}$ due to the mutation mechanism consists of parameter vector $\mathrm{X}_{j}^{k}$, the best particle $X_{\text {best }}^{k}$, and two randomly selected vectors. As comparison, the mutant vector $\mathrm{V}_{j}^{k+1}$ is generated according to Equation 12 for typical DE [31]: 


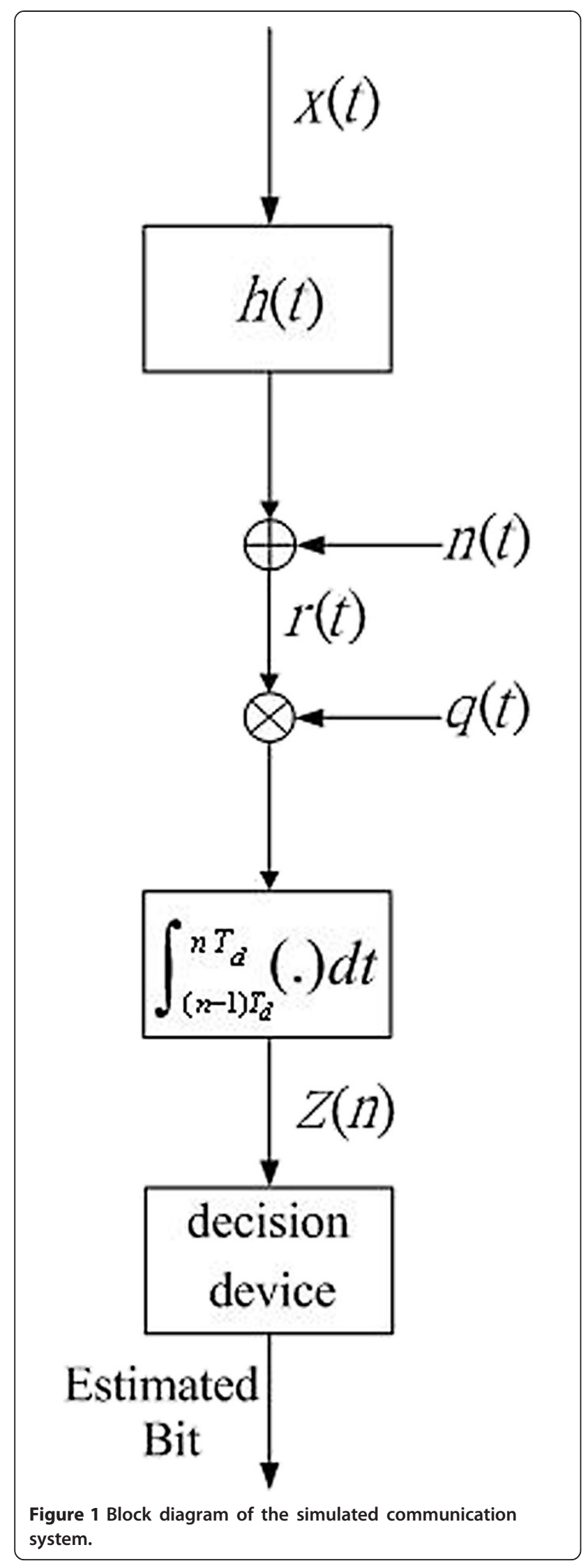

$$
\begin{aligned}
& \left(\mathrm{V}_{j}^{k+1}\right) i=\left(\mathrm{X}_{j}^{k}\right) i+\chi \cdot\left[\left(\mathrm{X}_{m}^{k}\right) i-\left(\mathrm{X}_{n}^{k}\right) i\right], \\
& j, m, n \in\left[0, N_{\mathrm{p}}-1\right], \quad m \neq n,
\end{aligned}
$$

where $i=1 \sim D$ and $\chi$ is the scaling factor associated with the vector difference $\left(X_{m}^{k}-X_{n}^{k}\right)$. Note that $\zeta$ is set to 0 for $\mathrm{DE}$; therefore, the main differences between DDE and DE is that DDE includes the idea of approaching the 'best' during the course of optimization procedure.

After mutation, the crossover operator is applied to generate another kind of new vector $\mathrm{u}_{j}$. The crossover operation in DDE delivers the crossover vector $\mathrm{u}_{j}^{k+1}$ by mixing the components of the current vector $\mathrm{X}_{i}$ and the above mutant vector $\mathrm{V}_{i}$. It can be expressed as

$$
\mathrm{u}_{j}^{k+1}=\left\{\begin{array}{c}
\left(\mathrm{V}_{j}^{k+1}\right)_{i}, Q_{k}<\mathrm{CR} \\
\left(\mathrm{X}_{j}^{k}\right)_{i}, Q_{k} \geq \mathrm{CR},
\end{array}\right.
$$

where $i=1 \sim D$ and $Q_{k}$ is a random number uniformly distributed within $[0,1] . C R \in(0,1)$ is a predefined crossover rate. DDE uses a greedy selection operator that is defined by

$$
\mathrm{X}_{j}^{k+1}=\left\{\begin{array}{cc}
\mathrm{u}_{j}^{k+1}, & \text { if } \mathrm{CF}\left(\mathrm{u}_{j}^{k}\right)<\mathrm{CF}\left(\mathrm{X}_{j}^{k}\right) \\
\mathrm{X}_{j}^{k}, & \text { otherwise. }
\end{array}\right.
$$

Selection operation is conducted by comparing the parent vector $\mathrm{X}_{j}^{k+1}$ with the crossover vector $\mathrm{u}_{j}^{k+1}$. The vector with smaller cost function $(\mathrm{CF})$ value is selected as a member for the next generation.

In the synthesis procedure, the DDE is used to minimize the following CF:

$$
\mathrm{CF}=\text { outage probability, }
$$

where cost function is the outage probability for UWB system. The outage probabilities for 100 -Mbps B-PAM and for a BER $<10^{-3}$ versus SNR are calculated. DDE is used to search the receiver antenna location to minimize the outage probability of the communication system. This SBR/image method technique is used to calculate the UWB channel impulse response for each location of the receiver. Based on the channel impulse response, the number of multipath components, the root mean square (RMS) delay spread $\tau_{\mathrm{RMS}}$, and the mean excess delay $\tau_{\mathrm{MED}}$ are computed. The DDE algorithm iteratively generates a new population that springs from the previous population through the application of the reproduction by mutation and replacement operators. In our simulation, when the cost function is bigger than the threshold 


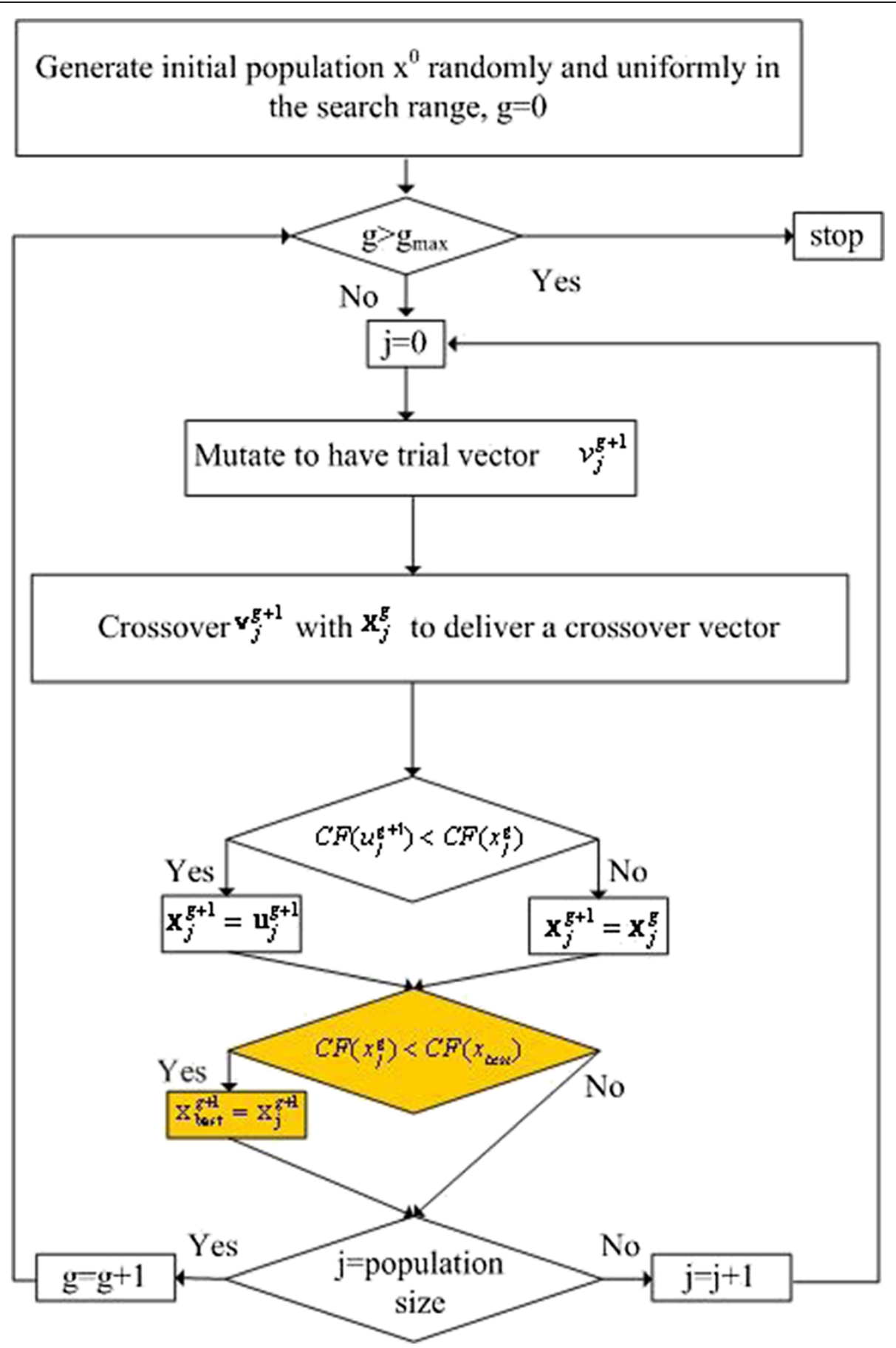

Figure $\mathbf{2}$ The flowchart of the dynamic differential evolution.

value or DDE does not find a better individual within 300 successive generations, the DDE will be terminated, and a solution is then obtained.

\subsection{Genetic algorithm}

Genetic algorithm (GA) is the global numerical optimization methods based on genetic recombination and evaluation in nature $[32,33]$. They use the iterative optimization procedures, which start with a randomly selected population of potential solutions and then gradually evolve toward a better solution through the application of the genetic operators. GA typically operates on a discretized and coded representation of the parameters rather than on the parameters themselves. These representations are often considered to be 
'chromosomes', while the individual element, which constitutes chromosomes, is the 'gene'. Simple but often very effective chromosome representations for optimization problem involving several continuous parameters can be obtained through the juxtaposition of discretized binary representations of the individual parameter.

We regulate the receiver antenna location of the indoor environment to minimize the outage probability. The GA starts with a population containing a total of $G_{\mathrm{P}}$ candidates (i.e., $G_{\mathrm{P}}$ is the population size). Each candidate is described by a chromosome. Then, the initial population can simply be created by taking $G_{\mathrm{P}}$ random chromosomes. GA iteratively generates a new population, which is derived from the previous population through the application of the reproduction, crossover, and mutation operators.

The GA is used to minimize the cost function in Equation 15, where cost function is the outage probability for UWB system. Through repeated applications of reproduction, crossover, and mutation operators, the initial population is transformed into a new population in an iterative manner. New populations will contain increasingly better chromosomes and will eventually converge to an optimal population that consists of the optimal chromosomes.
As for the GA calculations, each chromosome is assigned information of the position of transmitter as the optimization variables. For each generated chromosome, the GA reruns the ray tracing program to calculate the BER for each receiver from the given location of transmitter provided by the chromosome. In our simulation, when the BER is lower than the threshold value or GA does not find a better individual within 300 successive generations, the GA will be terminated and a solution is then obtained.

\section{Numerical results}

A ray tracing technique is developed to calculate the channel impulse response from 3.1 to $10.6 \mathrm{GHz}$ with frequency interval of $5 \mathrm{MHz}$, i.e., 1,501 frequency components are used. Since the dielectric constant and conductivity of the materials change with frequency, the different values of dielectric constant and conductivity of materials for different frequencies are carefully considered in channel calculation [34-37]. The BER for different receiver positions in the indoor environments are investigated. Figure 3 is the top view of the indoor environment with dimensions of $10 \mathrm{~m}$ (length) $\times 9.2 \mathrm{~m}$ (width) $\times 3 \mathrm{~m}$ (height). The transmitting and receiving antennas are with simple omnidirectional radiation pattern and vertically

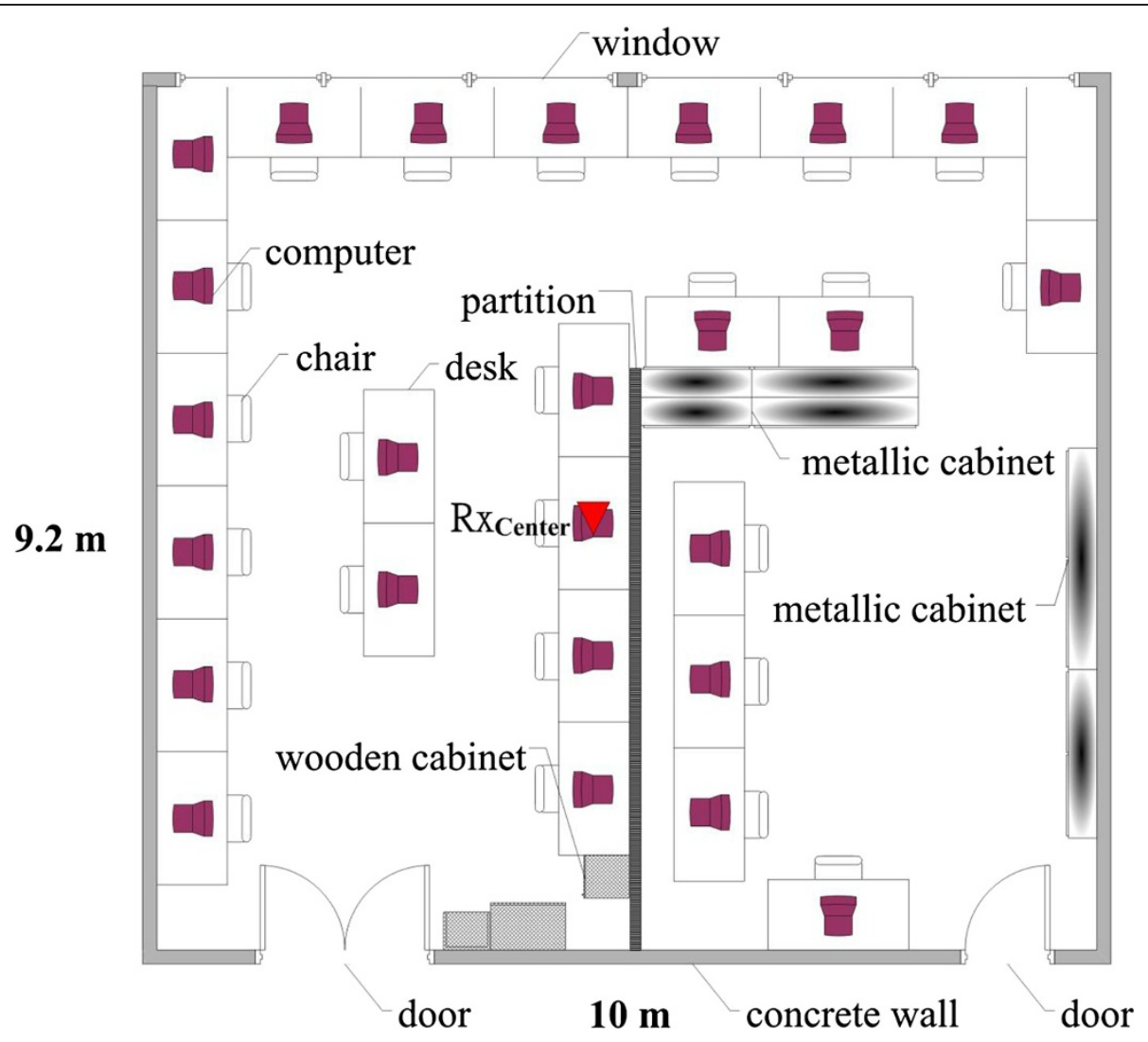

Figure 3 A plan view of the simulated environment. 


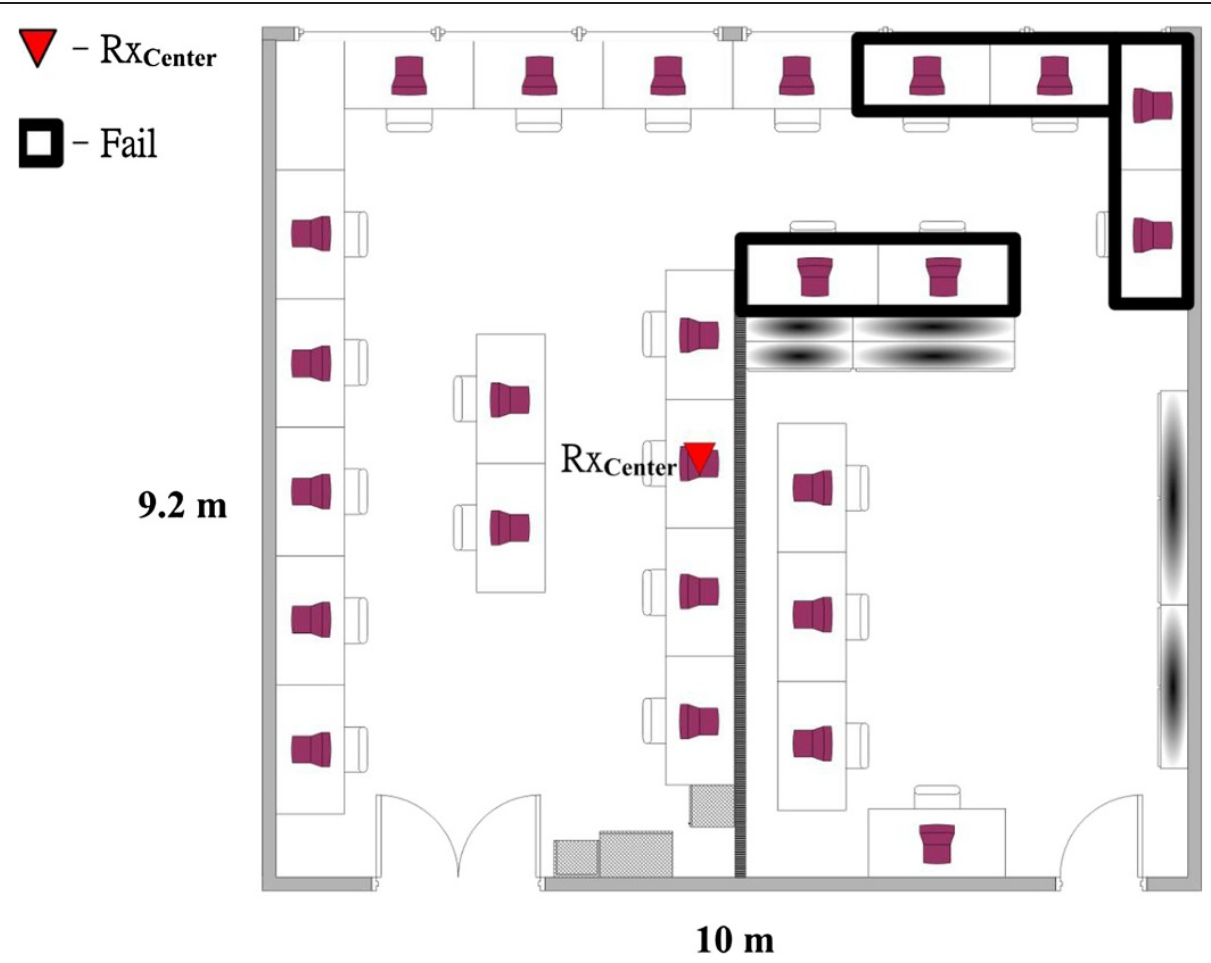

Figure 4 Outage areas $\left(B E R>10^{-3}\right)$ with the use of one transmitter.

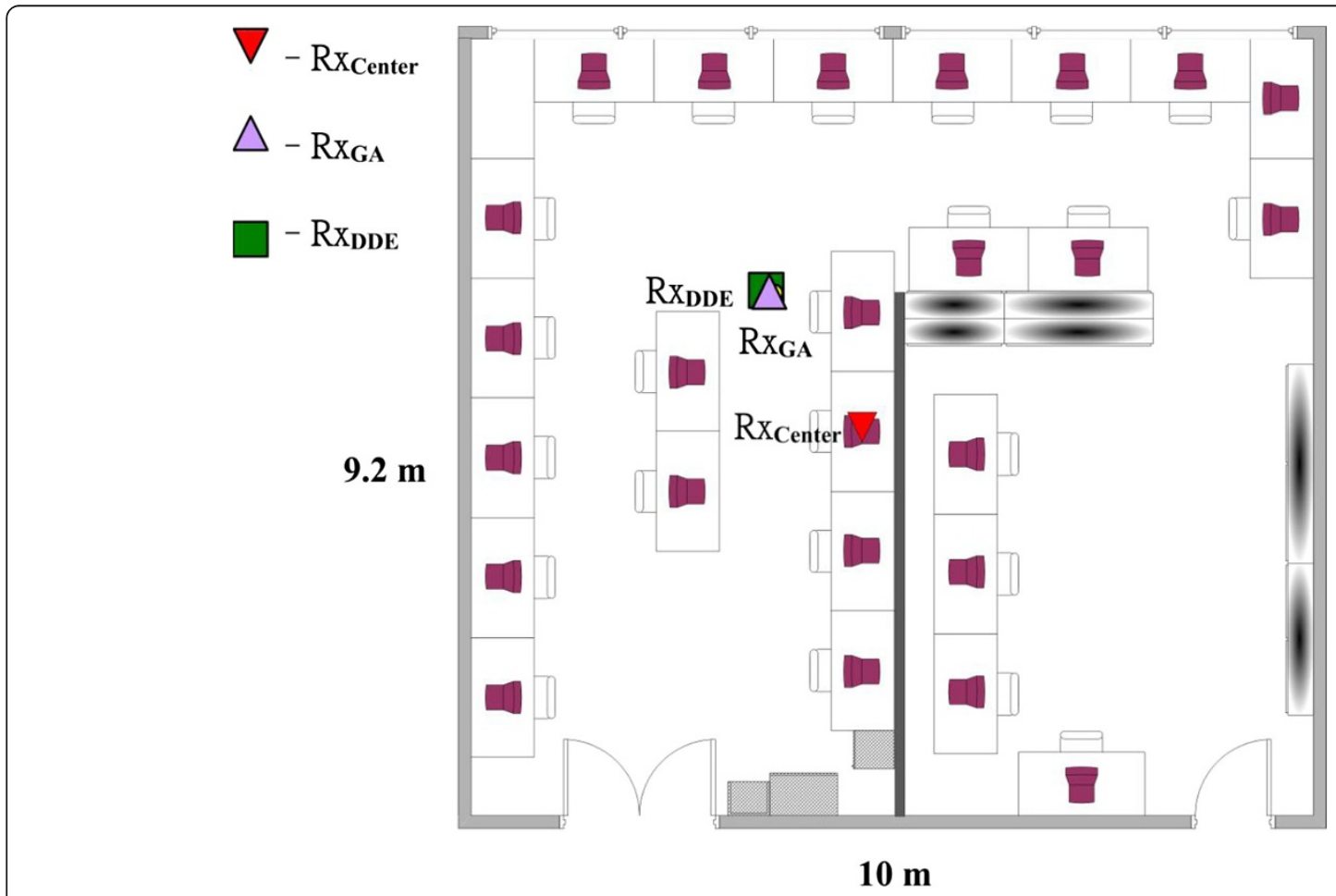

Figure 5 Optimized receiver position when only one receiver is used. $(\boldsymbol{\nabla})$ Receiver position $R x_{\text {Center }}$ is located in the center of the indoor

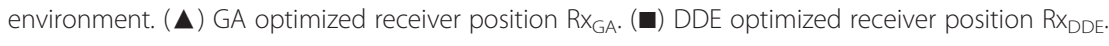


polarized. Each antenna is with a fixed height of $1 \mathrm{~m}$. The optimal antenna location for minimizing the outage probability is searched by DDE and GA. The relative coefficients of the DDE are set as follows: The scaling factors of $F$ and $\lambda$ are both 0.8 , and the crossover rate $C R=0.6$. The population size $N_{\mathrm{p}}=30$. The relative coefficients of the GA are set as follows: The crossover rate $G_{\mathrm{r}}=0.6$. The mutation probability $G_{M}=0.025$, and the population size $G_{\mathrm{P}}=30$. The cases with different transceiver positions are considered in our simulation. The receiving antenna $\mathrm{Rx}_{\text {Center }}(5 \mathrm{~m} \times$ $4.6 \mathrm{~m} \times 0.73 \mathrm{~m}$ ) located in the center of the indoor environment with a fixed height of $1 \mathrm{~m}$ is used for our reference, as shown in Figure 3.

Based on the channel impulse response, the number of multipath components, the RMS delay spread $\tau_{\text {RMS }}$, and the mean excess delay $\tau_{\text {MED }}$ are computed. The cumulative distributions of the RMS delay spread $\tau_{\mathrm{RMS}}$ is defined as [38]

$$
\tau_{\mathrm{RMS}}=\sqrt{\frac{\sum_{n=1}^{N} \tau_{n}{ }^{2}\left|a_{n}\right|^{2}}{G}-\left(\frac{\sum_{n=1}^{N} \tau_{n}\left|a_{n}\right|^{2}}{G}\right)^{2}},
$$

where $G=\sum_{n=1}^{N}\left|a_{n}\right|^{2}$ is the total multipath gain.

The mean excess delay, $\tau_{\mathrm{MED}}$, is defined as [39]

$$
\tau_{\mathrm{MED}}=\frac{\sum_{n=1}^{N} \tau_{n}\left|a_{n}\right|^{2}}{G} .
$$

By Equations 16 and 17, we can obtain the RMS delay spreads and mean excess delay.

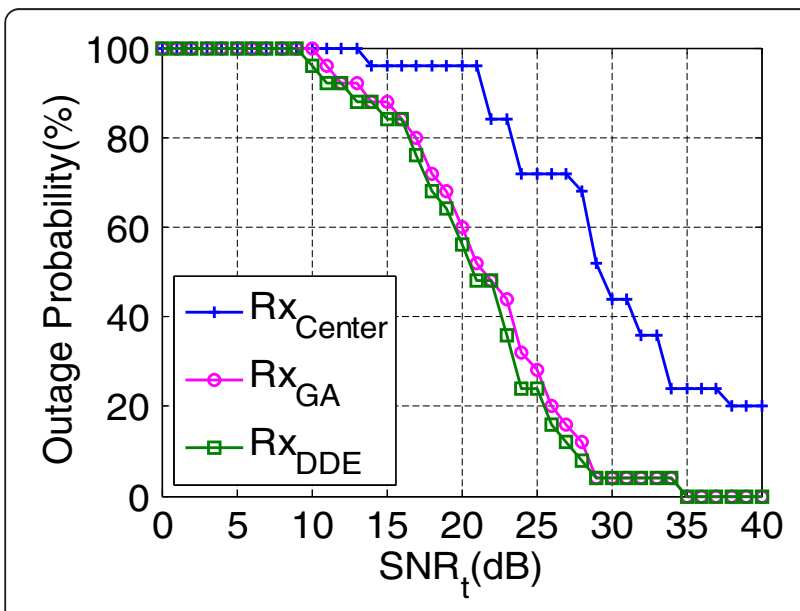

Figure 6 Outage probability versus SNR for the two kinds of algorithms.

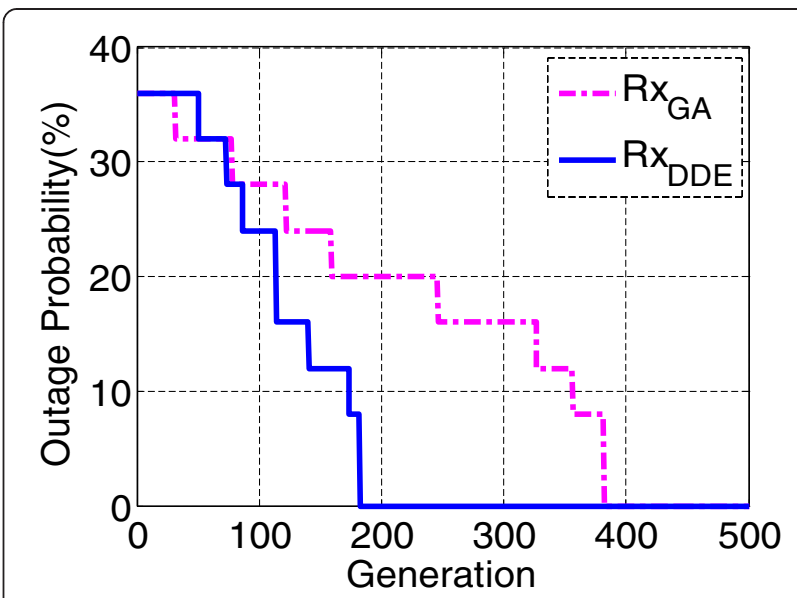

Figure 7 Average outage probability versus generations by two different algorithms.

We compare the effects of $\mathrm{Rx}_{\mathrm{Center}} \mathrm{Rx}_{\mathrm{DDE}}$, and $\mathrm{Rx}_{\mathrm{GA}}$ on UWB transmission. For the case, the receiver is mobile in the whole indoor environment and the transceivers are located on the desk, in which 25 measurements are carried out (e.g., transmission from a computer ' $T x$ ' to a printer ' $R x^{\prime}$ ). Figure 4 shows that the transmitting antenna $\operatorname{Rx}_{\text {Center }}(5 \mathrm{~m} \times 4.6 \mathrm{~m} \times 0.73 \mathrm{~m})$ is located in the center of the indoor environment. In this case, six bad receiving signal points $\left(\mathrm{BER}>10^{-3}\right.$ ) occurred including (not highlighted in the figure) the transmitter points. The DDE and GA optimization position of the receiver is shown in Figure 5. The optimal locations of the receiving antennas are at $\operatorname{Rx}_{\mathrm{DDE}}(3.52 \mathrm{~m} \times 6.25 \mathrm{~m} \times 0.73 \mathrm{~m})$ and $\mathrm{Rx}_{\mathrm{GA}}$ $(3.56 \mathrm{~m} \times 6.24 \mathrm{~m} \times 0.73 \mathrm{~m})$ using the DDE and GA, respectively. The optimization receiver points $\mathrm{Rx}_{\mathrm{DDE}}$ and $\mathrm{Rx}_{\mathrm{GA}}$ are chosen as the receiver locations as squares/triangle, and no bad transmitting signal point exists. The outage probability versus SNR is calculated, as shown in Figure 6. Here, SNR is defined as the ratio of the average transmitted power to the noise power at the front end of the receiver. It is seen that the outage probability at SNR = $28 \mathrm{~dB}$ are about $68 \%, 8 \%$, and $12 \%$ for $\mathrm{Rx}_{\mathrm{Centen}} \mathrm{Rx}_{\mathrm{DDE}}$, and $\mathrm{Rx}_{\mathrm{GA}}$, respectively. It is clear that the outage probability decreased by DDE is better than those by GA about $50 \%$. The performance for the antenna location by DDE is better than that by GA.

Table 1 The channel parameters ( $\tau_{\text {MED }}$ and $\tau_{\text {RMS }}$ ) for the two kinds of algorithms

\begin{tabular}{lcc}
\hline & \multicolumn{2}{c}{ Transmitter channel parameters } \\
\cline { 2 - 3 } & $\boldsymbol{\tau}_{\text {MED }}$ (ns) & $\boldsymbol{\tau}_{\text {RMS }}$ (ns) \\
\hline $\mathrm{Rx}_{\text {Center }}$ & 7.64 & 12.50 \\
$\mathrm{Rx}_{\mathrm{GA}}$ & 2.86 & 7.12 \\
$\mathrm{Rx}_{\mathrm{DDE}}$ & 2.80 & 6.69 \\
\hline
\end{tabular}


The outage probability versus generation DDE and GA are calculated, as shown in Figure 7 . It is found that the outage probability decreases quickly by the DDE, and good convergences are achieved within 182 generations. It also shows that the convergent speed for the DDE is faster than that for GA.

In order to determine the multipath effect, the $\tau_{\mathrm{RMS}}$ and $\tau_{\text {MED }}$ were calculated. A summary of these values are given in Table 1 for scenarios. For the transceivers located on the table's case scenario, the $\tau_{\mathrm{RMS}}$ for $\mathrm{Rx}_{\text {Center }}$ $\mathrm{Rx}_{\mathrm{DDE}}$, and $\mathrm{Rx}_{\mathrm{GA}}$ are $12.5,2.80$, and $2.86 \mathrm{~ns}$, respectively. It is clear that the $\tau_{\mathrm{RMS}}$ for the $\mathrm{Rx}_{\mathrm{Center}}$ case is the biggest.

\section{Conclusions}

Using the impulse responses of these multipath channels, the BER performance for UWB communication system is calculated. The DDE and GA are used to minimize the BER. Numerical results show that BER in the cases can be decreased. It is also found that the BER decreased by DDE is better than that by GA. Numerical results also show that the DDE outperforms the GA in convergence speed.

\section{Competing interests}

The authors declare that they have no competing interests.

\section{Author details}

'Department of Electrical Engineering, Tamkang University, Tamsui District, New Taipei City 251, Taiwan. ${ }^{2}$ Department of Computer and Communication Engineering, Taipei College of Maritime Technology, Danshui Town, New Taipei City 251, Taiwan. ${ }^{3}$ Peter Kiewit Institute, University of Nebraska-Lincoln, Lincoln, NE 68588, USA.

\section{Received: 8 May 2013 Accepted: 3 September 2013}

Published: 25 September 2013

\section{References}

1. Federal Communications Commission, Revision of part 15 of the commission's rules regarding ultra-wideband transmission systems. First report and order (FCC, Washington, DC, 2002)

2. AH Wong, MJ Neve, KW Sowerby, Antenna selection and deployment strategies for indoor wireless communication systems. IET Commun. 1(4), 732-738 (2007)

3. DCK Lee, MJ Neve, KW Sowerby, The impact of structural shielding on the performance of wireless systems in a single-floor office building. IEEE Trans. on Wireless Comm. 6(5), 1787-1795 (2007)

4. CC Chiu, CL Liu, SH Liao, Channel characteristics of ultra-wideband systems with single co-channel interference. Wirel. Commun. Mob. Comput. 13(9), 864-873 (2013)

5. CC Chiu, CY Yu, SH Liao, MK Wu, Channel capacity of multiple-input multiple-output systems for optimal antenna spacing by particle swarm optimizer. Wirel. Pers. Commun. 69(4), 1865-1876 (2013)

6. CC Chiu, CH Chen, SH Liao, KC Chen, Bit error rate reduction by smart UWB antenna array in indoor wireless communication. J. Appl. Sci. Eng. 15(2), 139-148 (2012)

7. CC Chiu, CH Chen, SH Liao, TC Tu, Ultra-wideband outdoor communication characteristics with and without traffic. EURASIP J. Wirel. Commun. Netw. 2012, 92 (2012)

8. CC Chiu, YT Kao, SH Liao, YF Huang, UWB communication characteristics for different materials and shapes of the stairs. J. Commun. 6(8), 628-632 (2011)

9. SH Liao, HP Chen, CC Chiu, CL Liu, Channel capacities of indoor MIMO-UWB transmission for different material partitions. Tamkang J. Sci. Eng. 14(1), 49-63 (2011)

10. CL Liu, CC Chiu, SH Liao, YS Chen, Impact of metallic furniture on UWB channel statistical characteristics. Tamkang J. Sci. Eng. 12(3), 271-278 (2009)
11. SH Liao, CC Chiu, MH Ho, Comparison of dynamic differential evolution and genetic algorithm for MIMO-WLAN transmitter antenna location in indoor environment. Wirel. Pers. Commun. 71(4), 2677-2691 (2013)

12. CC Chiu, MH Ho, SH Liao, MIMO-UWB smart antenna communication characteristics for different antenna arrays of transmitters. Int. J. RF and Microwave Computer-Aided Eng. 23(3), 378-392 (2013)

13. CC Chiu, MH Ho, SH Liao, PSO and APSO for optimizing coverage in indoor UWB communication system. Int. J. RF and Microwave Computer-Aided Eng. 23(3), 300-308 (2013)

14. SH Liao, CC Chiu, $\mathrm{CH}$ Chen, MH Ho, Channel characteristics of MIMO-WLAN communications at $60 \mathrm{GHz}$ for various corridors. EURASIP J. Wirel. Commun. Netw. 2013, 96 (2013)

15. $\mathrm{MH} \mathrm{Ho}, \mathrm{CC}$ Chiu, SH Liao, Comparison of different antenna arrays for the BER reduction in indoor wireless communication. Int. J. Commun. Syst. 26(2), 161-176 (2013)

16. CH Sun, CC Chiu, MH Ho, CL Li, Comparison of dynamic differential evolution and self-adaptive dynamic differential evolution for buried metallic cylinder. Res. Nondestruct. Eval. 24, 35-50 (2013)

17. $\mathrm{MH} \mathrm{Ho}, \mathrm{CC}$ Chiu, SH Liao, Optimization of channel capacity for MIMO smart antenna using particle swarm optimizer. IET Commun. 6(16), 2645-2653 (2012)

18. $\mathrm{MH} \mathrm{Ho}, \mathrm{CC}$ Chiu, SH Liao, Bit error rate reduction for circular ultrawideband antenna by dynamic differential evolution. Int. J. RF and Microwave Computer-Aided Eng. 22(2), 260-271 (2012)

19. SH Liao, MH Ho, CC Chiu, CH Lin, Optimal relay antenna location in indoor environment using particle swarm optimizer and genetic algorithm. Wirel. Pers. Commun. 62(3), 599-615 (2012)

20. SH Liao, CC Chiu, MH Ho, CL Liu, Channel capacity of multiple-input multiple-output ultra wide band systems with single co-channel interference. Int. J. Commun. Syst. 23(12), 1600-1612 (2010)

21. $\mathrm{SH}$ Liao, $\mathrm{MH} \mathrm{Ho}, \mathrm{CC}$ Chiu, Bit error rate reduction for multiusers by smart UWB antenna array. Prog. Electromagn. Res.C 16, 85-98 (2010)

22. $\mathrm{MH} \mathrm{Ho}, \mathrm{SH}$ Liao, CC Chiu, UWB communication characteristics for different distribution of people and various materials of walls. Tamkang J. Sci. Eng. 13(3), 315-326 (2010)

23. $\mathrm{MH}$ Ho, SH Liao, CC Chiu, A novel smart UWB antenna array design by PSO. Prog. Electromagn. Res.C 15, 103-115 (2010)

24. SH Chen, SK Jeng, An SBR/image approach for indoor radio propagation in a corridor. IEICE Trans. Electron E78-C, 1058-1062 (1995)

25. SH Chen, SK Jeng, SBR/image approach for indoor radio propagation in tunnels with and without traffic. IEEE Trans. Veh. Technol. 45, 570-578 (1996)

26. I Oppermann, M Hamalainen, J linatti, UWB Theory and Applications (Wiley, New York, 2004)

27. EW Kamen, BS Heck, Fundamentals of Signals and Systems Using the Web and MATLAB (Upper Saddle River, Prentice Hall, 2000)

28. T Zhi, GB Giannakis, BER sensitivity to mistiming in ultra-wideband impulse radios-part I: nonrandom channels. IEEE Trans. Signal Process. 53, 1550-1560

29. EA Homier, RA Scholtz, Rapid acquisition of ultra-wideband signals in the dense multipath channel. IEEE Conference on Ultra Wideband System and Technologies, Baltimore, May 2002 (IEEE, Piscataway, 2002), pp. 105-109

30. DJ Gargin, A fast and reliable acquisition scheme for detecting ultra wide-band impulse radio signals in the presence of multi-path and multiple access interference, International Workshop on Ultra Wideband System, May 2004 (Piscataway, IEEE, 2004). pp. 106-110

31. R Storn, K Price, Differential evolution - a simple and efficient adaptive scheme for global optimization over continuous spaces, Technical report TR-95-012 (International Computer Science Institute, Berkeley, 1995)

32. DE Goldberg, Genetic Algorithm in Search, Optimization and Machine Learning (Addison-Wesley, Boston, 1989)

33. J Michael Johnson, Y Rahmat-Samii, Genetic algorithms in engineering electromagnetics. IEEE Antenn. Propag. Mag. 39(4), 7-21 (1997)

34. Y Zhao, Y Hao, A Akram, P Clive, UWB on-body radio channel modeling using ray theory and subband FDTD method. IEEE Trans. Microw. Theor. Tech. 54, 1827-1835 (2006)

35. RM Buehrer, A Safaai-Jazi, W Davis, D Sweeney, Ultra-wideband propagation measurements and modeling. Final report, DARPA NETEX Program Virginia Tech, 3rd edn., 2004, pp. 38-216

36. A Muqaibel, A Safaai-Jazi, A Bayram, AM Attiya, SM Riad, Ultra-wideband through-the-wall propagation. IEEE Proceedings Microwaves, Antennas and Propagation, December 2005 (IEEE, Piscataway, NJ, 2005), pp. 581-588 
37. AS Jazi, SM Riad, A Muqaibel, A Bayram, Through-the-wall propagation and material characterization, DARPA NETEX Program Report, 2002

38. M Gabriella, D Benedetto, G Giancola, Understanding Ultra Wide Band Radio Fundamentals (Prentice Hall, Upper Saddle River, 2004)

39. TS Rappaport, Wireless Communications: Principles and Practice, 2nd edn. (Upper Saddle River, Prentice Hall, 2002)

doi:10.1186/1687-1499-2013-235

Cite this article as: Liao et al:: Optimal receiver antenna location in indoor environment using dynamic differential evolution and genetic algorithm. EURASIP Journal on Wireless Communications and Networking $20132013: 235$

Submit your manuscript to a SpringerOpen ${ }^{\circ}$ journal and benefit from:

- Convenient online submission

- Rigorous peer review

- Immediate publication on acceptance

- Open access: articles freely available online

- High visibility within the field

- Retaining the copyright to your article 